\title{
Coherent photon beam based diagnostics for a seeded extreme ultraviolet free-electron laser
}

\author{
Chao Feng, Haixiao Deng, Zhimin Dai, Bo Liu, Shunqiang Tian, \\ Manzhou Zhang, and Meng Zhang \\ Shanghai Institute of Applied Physics, Chinese Academy of Sciences, Shanghai 201800, China
}

(Received 9 December 2013; published 14 October 2014)

\begin{abstract}
Independently from conventional electron beam based procedures, the photon beam based diagnostic is an alternative way for electron trajectory alignment and commissioning of numerous undulator cells in high-gain short-wavelength free-electron lasers (FELs). In this paper, using the microbunched electron beam and the undulator fine-tuning technique, a novel method based on the spatial profile analysis of coherent harmonic radiation from individual or two consecutive undulator segments, the so-called coherent photon beam based diagnostic, is proposed for undulator alignment and commissioning in seeded FELs. The preliminary experimental results at the Shanghai deep ultraviolet FEL test facility are presented. It shows that the proposed method is effective and convenient in the electron beam trajectory control, the undulator magnetic gap verification, and the phase match between two undulator segments.
\end{abstract}

\section{INTRODUCTION}

With the great success of $\mathrm{x}$-ray free-electron laser (FEL) facilities all over the world [1-4], scientists now have the ability to investigate the matter with femtosecond x-ray single-shot diffraction [5] and time-resolved pump-probe experiments [6]. Therefore, more and more FEL user facilities are under construction or being proposed, from the extreme ultraviolet [7-10] to hard x-ray [11-13] spectral region. Generally, the undulator system of a short-wavelength FEL is made up of periodic undulator modules, which consists of an undulator segment and an insert section containing various items such as beam position monitors, quadrupoles, phase shifters, steering coils and vacuum components. As well known, the FEL exponential gain requires stringent electron beam trajectory control along the whole undulator system, fine undulator magnetic field setting and well-matched phase between two consecutive undulator segments. For this purpose, the electron beam based alignment [14-16] has been developed. While the electron beam based alignment has already great success in X-ray FELs [1], it shows no insight into the undulator magnetic gap or the phase match of adjacent undulator segments.

In general, "photon diagnostics" such as optical transition radiation screen, FEL intensity monitor, FEL spectrometers and terahertz streaking, etc., are widely used

\footnotetext{
*Corresponding author. denghaixiao@sinap.ac.cn

Published by the American Physical Society under the terms of the Creative Commons Attribution 3.0 License. Further distribution of this work must maintain attribution to the author $(s)$ and the published article's title, journal citation, and DOI.
}

in FEL facilities; in the following discussions, it is restricted to the undulator alignment and commissioning. Independently from the electron beam based alignment, the photon beam based diagnostic [17] is a complementary tool for alignment and commissioning of the FEL undulator modules, which has been currently used at the SACLA user facility [18], and was seriously considered by the European-XFEL project [19]. In the photon beam based procedure, the photon instruments consisting of a monochromator, an imaging optics and a photon detector are located downstream of the undulator system, and thus allow one to characterize the spontaneous emission (SE) of the selected undulator segment and enable a precise alignment and setup of the whole undulator system. However, to the best of our knowledge, photon beam based diagnostics has only been considered for self-amplified spontaneous emission (SASE) [20-22] based x-ray FELs. In recent years, several seeded FEL facilities have been constructed or proposed from the extreme ultraviolet [4,23-26] to the $\mathrm{x}$-ray region [5-7,27,28]. Comparing with a SASE FEL, seeding schemes have the advantage in monochromic light generation with the help of various laser-beam interactions [29-32] or direct amplification of a coherent seed source $[24,33,34]$. In this paper, using the undulator radiation generated by the microbunched electron beam, a coherent photon beam based method is proposed for alignment and commissioning of the undulator system in seeded FELs. It is demonstrated that the coherent photon beam based method is much easier to be realized on seeded FELs without utilization of a monochromator, when compared with the conventional photon beam based procedures $[17,18]$.

We first describe the principle of the coherent photon beam based method to optimize the electron beam trajectory, to verify the undulator magnetic gap, and to adjust the 
phase match between two successive undulator segments in Sec. II. On the basis of the Dalian coherent light source (DCLS) [7], numerical simulations are given to demonstrate this method in Sec. III. Finally, the preliminary experimental results at the Shanghai deep ultraviolet FEL test facility (SDUV-FEL) [35] are presented in Sec. IV.

\section{COHERENT PHOTON BEAM BASED METHOD}

In this section, we illustrate the principle of coherent photon beam based diagnostics using the main parameters of DCLS [7] which is an extreme ultraviolet high-gain harmonic generation (HGHG) [29] FEL user facility under construction in China. The schematic of the DCLS layout is shown in Fig. 1. The linac of DCLS will provide electron beam with beam energy up to $300 \mathrm{MeV}$, bunch charge of about $500 \mathrm{pC}$, pulse duration of around $1.6 \mathrm{ps}$, and normalized emittance lower than $1 \mathrm{~mm}$ mrad. The undulator system consists of one segment of modulator and four segments of radiator, where the period length is 50 and $30 \mathrm{~mm}$, respectively. A seed laser with pulse length of around 1 ps (FWHM) is employed to interact with the electron beam in the modulator. Then with the combination of optical parametrical amplification laser technique (i.e., 240 to $360 \mathrm{~nm}$ seed laser), variable gap undulator (i.e., 9 to $18 \mathrm{~mm}$ alterable gap radiator) and harmonic selection (i.e., from the 2 nd harmonic to the 5 th harmonic), DCLS is able to generate fully coherent FEL radiation with continuous tuning ability over $50-150 \mathrm{~nm}$ by a single stage HGHG. However, for simplification in the following calculations, only the $50 \mathrm{~nm}$ case is considered.

One of the key issues for operating HGHG is to achieve temporal and spatial overlap of the electron bunches and the external seed laser pulses in the modulator. The transverse overlap can be achieved by observing the electron beam and the laser beam on yttrium-aluminum-garnet screens located upstream and downstream of the modulator. The spontaneous emission from the electron beam and the seed laser pulse are sent to a fast photodiode to find the initial coarse temporal overlap. Then the exact temporal overlap can be obtained by fine-tuning the delay line of the seed laser. The overlap between the seed pulse and electron beam in the modulator helps one to get sufficient energy modulation in the electron beam, which will be converted to longitudinal density modulation by a small dispersive chicane. After that, the electron beam with strong microbunching will emit intense coherent high harmonic radiation in the radiator undulator. In the first two gain lengths of the radiator, FEL works in the coherent harmonic generation regime $(\mathrm{CHG})$ [29], where the harmonic field grows linearly with the radiator length $z$. Thus, CHG intensity is much higher than spontaneous emission even at the very beginning of the radiator. The intensity analysis of such coherent photon beam can be used to roughly estimate the temporal structure of the electron beam current and emittance [36], or precisely determine the averaged slice energy spread [37,38], while spatial profile studies, as we will show below, will allow one to optimize the electron beam trajectory, verify the undulator magnetic gap, and adjust the phase match between two undulator segments.

The comparison of different undulator commissioning methods is given in Table I. The electron beam based alignment [14-16] has been successfully operated at LCLS. However, it cannot be used for optimization of undulator magnetic gap, phase match of adjacent undulator segments or taper of the entire undulator. The basic idea of photon beam based undulator commissioning in a SASE FEL is spectral or spatial analysis of the spontaneous emission from undulators, where a crystal monochromator is utilized for accurate spectrum selection with respect to the undulator resonant wavelength. In a seeded FEL configuration, because of the laser induced coherent microbunching, only a relatively narrow part within the spontaneous emission bandwidth is significantly amplified in radiator undulator. Therefore, the coherent photon beam based method can be accomplished without a monochromator, which will greatly simplify the experimental setup. Figure 2 gives the simulated spectra of coherent radiation and spontaneous emission after one undulator segment. Since the electron beam is strongly bunched at $50 \mathrm{~nm}$, the coherent undulator radiation results in a clean spectrum at $50 \mathrm{~nm}$, while the undulator resonant wavelength is changed in a reasonable range by tuning the undulator gap.

According to the undulator radiation physics, the spectral power per unit solid angle of a single electron in an undulator leads to the following expressions [40]:

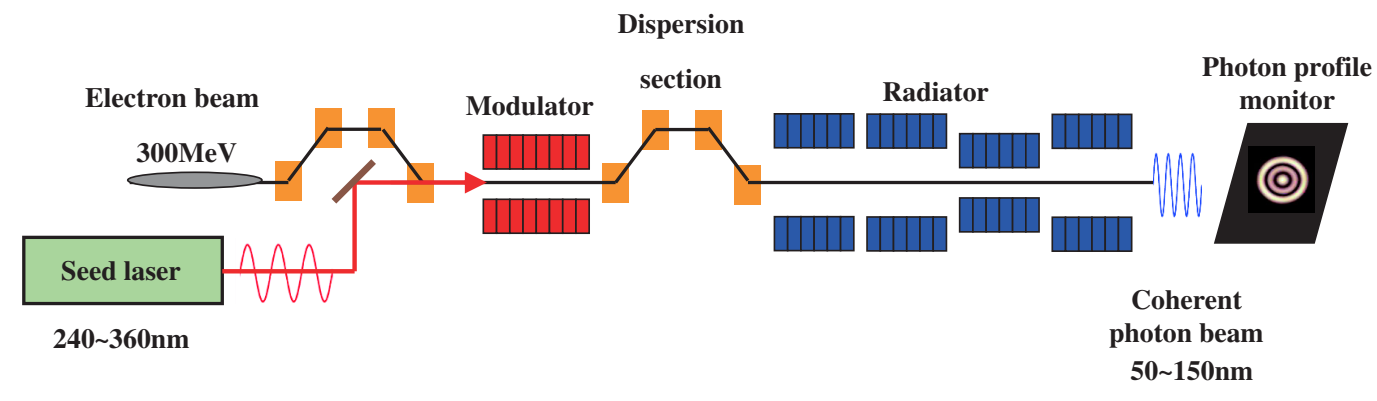

FIG. 1. Schematic of a seeded extreme ultraviolet FEL. 
TABLE I. Comparisons of different undulator commissioning methods.

\begin{tabular}{lcccccc}
\hline \hline & & \multicolumn{2}{c}{ Photon beam based method } & & \multicolumn{2}{c}{ Coherent photon beam based method } \\
\cline { 3 - 4 } Commissioning target & Beam based method & Radiation type & Item & & Radiation type \\
\hline Trajectory & Beam position & Monochromic SE & Angular profile & & CHG & Angular profile \\
K value & Not suitable & Monochromic SE & Spectrum & & CHG & Angular profile \\
Phase matching & Not suitable & Mono. SASE & Spectrum & & CHG & Angular profile \\
Undulator taper & Not suitable & SASE & Intensity & & CHG & Angular profile \\
\hline \hline
\end{tabular}

$$
\begin{gathered}
\frac{\partial^{2} P}{\partial \lambda \partial \Omega} \propto\left(\frac{\sin \left(\frac{\lambda-\lambda_{R}}{\lambda_{R}} \pi N\right)}{\frac{\lambda-\lambda_{R}}{\lambda_{R}} \pi N}\right)^{2}, \\
\lambda_{R}=\frac{\lambda_{u}\left(1+K^{2} / 2+\gamma^{2} \theta^{2}\right)}{2 \gamma^{2}}, \\
K \approx 0.934\left(1+\frac{2 \pi^{2} y^{2}}{\lambda_{u}{ }^{2}}\right) B_{0}[T] \lambda_{u}[\mathrm{~cm}],
\end{gathered}
$$

where $\lambda_{R}$ is the fundamental resonant wavelength of the undulator radiation, $N$ is the number of undulator period, $\lambda_{u}$ is the undulator period length, $K$ is the normalized undulator parameter, $\gamma$ is the kinetic energy of the electrons measured in units of its rest mass, $\theta$ is the observation angle, and $B_{0}$ is the peak magnetic field on the undulator axis.

While the electron beam is bunched at $50 \mathrm{~nm}$, Fig. 3 displays the normalized transverse distributions of coherent photon beam for slightly tuning the undulator gap, where

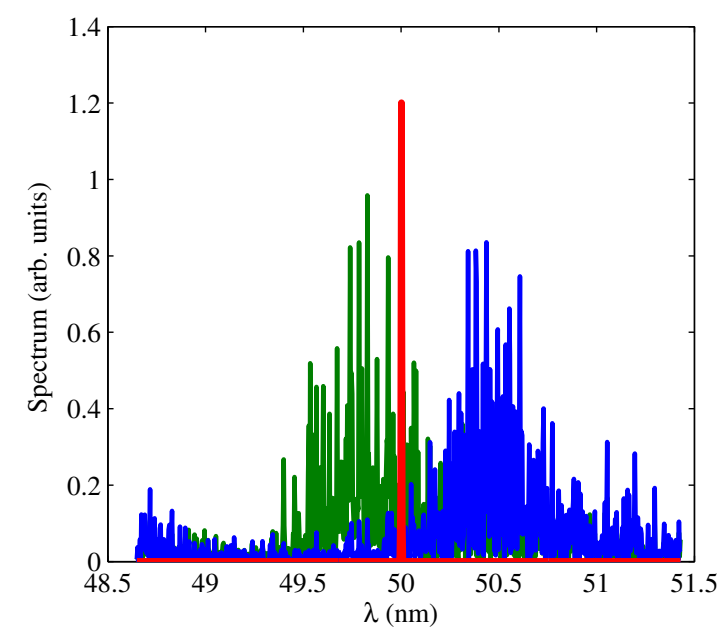

FIG. 2. The red line represents the coherent photon beam from undulator, while others represent the undulator spontaneous emission. All spectra are from GENESIS [39] simulations, where two undulator resonant wavelengths $\lambda_{R}=49.7 \mathrm{~nm}$ (green line) and $\lambda_{R}=50.5 \mathrm{~nm}$ (blue line) are used. The central wavelength of coherent photon beam is unchanged for different undulator resonant wavelengths. The real amplitude of the red is $\sim 10^{3}$ larger than the blue and green. all calculations have been performed for an observation point $3 \mathrm{~m}$ downstream the undulator. The coherent photon power scan suggests an optimized radiator undulator resonant wavelength of $49.84 \mathrm{~nm}$. This small discrepancy is due to the undulator detuning effect. It is clear that a Gaussian spatial mode is observed when the coherent photon power is optimized. For undulator resonant wavelength shorter than $49.84 \mathrm{~nm}$, a broadening accompanied by a splitting of the intensity cone towards rings is obtained. Detuning the undulator resonant wavelength by $0.36 \mathrm{~nm}$ longer will lead to a considerable narrowing of the radiation cone. These spatial distributions of coherent photon beam can be intensively analyzed with Eqs. (1) to (3), and thus used for electron trajectory alignment and commissioning of the FEL undulator modules.

\section{NUMERICAL CALCULATIONS FOR DCLS}

In this section, a numerical example of the coherent photon beam based method is illustrated on the basis of DCLS with the parameters given in Sec. II. Figure 4 shows the start-to-end simulation results of DCLS. The electron beam dynamics in a photoinjector was simulated with ASTRA [41] to take into account space-charge effects. ELEGANT [42] was then used for the simulation in the remainder of the linac. The FEL performance was simulated with GENESIS based on the output of ELEGANT. It is clearly seen that the $50 \mathrm{~nm}$ FEL radiation performs good temporal coherence and powerful output pulse energy. In some of our simulations, the undulator taper technique [43-45] is employed for the last two undulator segments, which enhances the final output pulse energy by a factor of about 2 [red line in Fig. 4(a)]. It means that the magnetic gap of six undulator segments should be independently tuned to the optimal.

\section{A. Optimize the magnetic gap of the undulator}

The coherent photon beam based diagnostic provides an opportunity to quickly optimize the magnetic gap of the radiator undulator. If we suppose an observation point $3 \mathrm{~m}$ after the final radiator undulator, gap tuning of six undulator segments can be accomplished in a successive way, i.e., always only one undulator gap is closed. Then the spatial distribution of coherent radiation at the observation point depends on the radiation undulator gap setting, as shown in Fig. 5. The coherent photon beam of the first four 

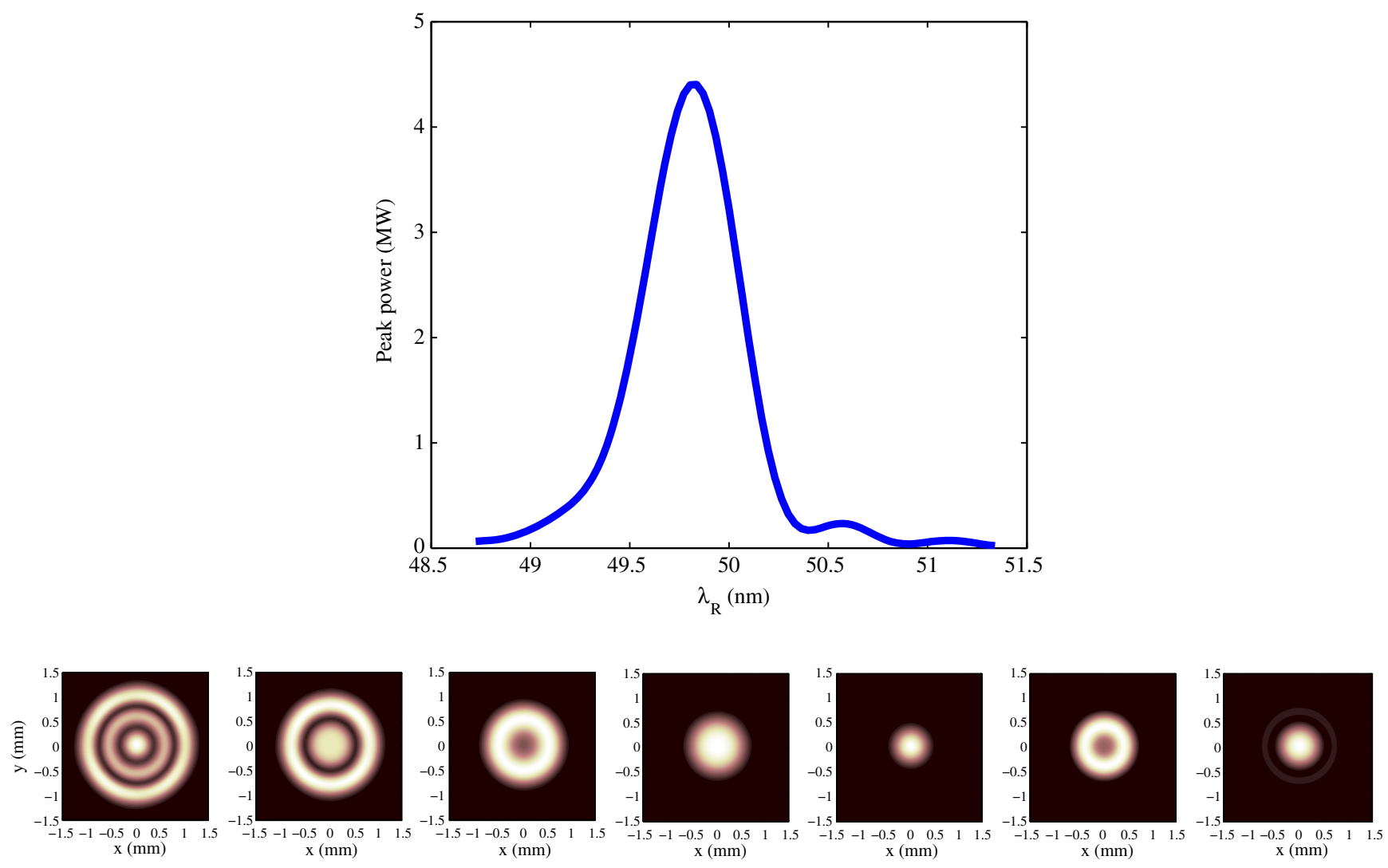

FIG. 3. The peak power (the upper plot) and the spatial profile (the lower plots) of coherent photon beam dependence on the radiator resonant wavelength (i.e., scanning the undulator gap). The radiator resonant wavelength for the lower plots is $48.84,49.14,49.49$, $49.84,50.20,50.47$, and $50.70 \mathrm{~nm}$ from left to right, respectively.
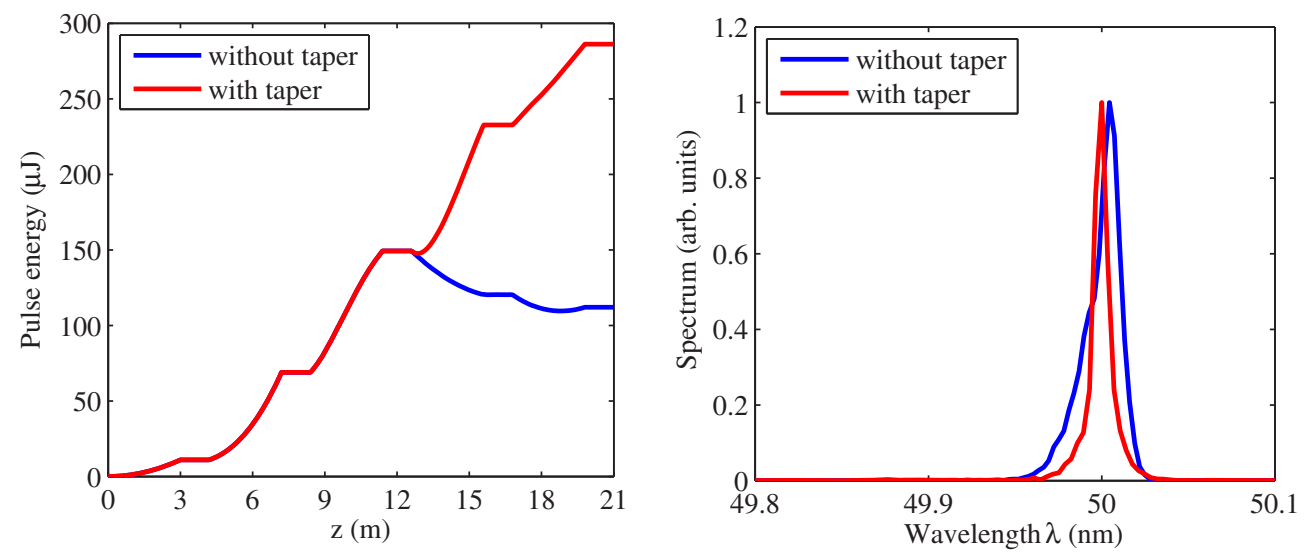

FIG. 4. Start-to-end simulation results for FEL peak power growth in the radiator undulator and the final output radiation spectrum of DCLS.

radiator undulator segments shows an ideal Gaussian spatial distribution, since the radiator undulator resonances of them are optimized for maximum output intensity. Meanwhile, because a stepped taper is applied to the last two radiator segments, i.e., the undulator resonant wavelength becomes shorter, a ring shape rather than core shape spatial distribution of coherent photon beam will be observed.

\section{B. Phase matching between two adjacent undulator segments}

Generally, to compensate the wavelength dependent phase condition when changing the undulator magnetic gap, a phase shifter is installed between two adjacent radiator segments. It consists of a two periods' undulator with gap-dependent magnetic field that delays the electron 

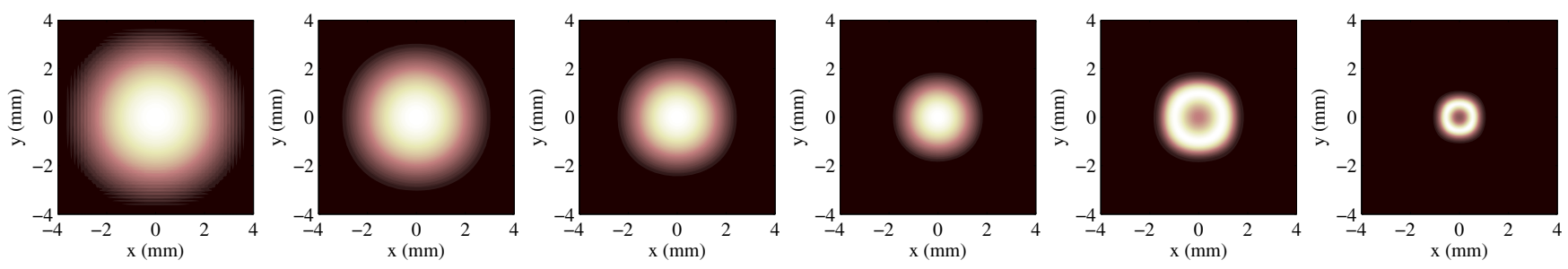

FIG. 5. Coherent photon beam spatial profile from six single radiator segments, where a stepped taper is applied to the last two radiator undulators for radiation power enhancement.

beam to match the phases between the radiation pulses from two adjacent undulator segments. The adjustment of the phase has to be assured with an accuracy of a few degrees for DCLS. The determination of the correct phase is based on observing the spatial distribution of the composed coherent photon beam of two successive radiator undulator segments (see Fig. 6). Here we consider two cases with undulator resonant wavelength of 49.49 and $49.84 \mathrm{~nm}$, respectively. For complete phase match, the spatial radiation distribution of the composed radiation is similar with the matched phase condition (upper), whereas the coherent photon beam is emitted in a ring in the fully destructively interfering case (lower).

\section{Alignment of electron beam trajectory}

Quadrupole offset will lead to a misalignment of electron beam trajectory along the undulator system, which may lead to mismatch of FEL resonance and transverse overlap between the electron beam and FEL radiation, and thus FEL gain reduction. For the DCLS, the FEL physics study shows an upper limit trajectory displacement of $20 \mu \mathrm{m}$ and $10 \mu \mathrm{rad}$ angular trajectory alignment requirement within a single undulator segment. While the electron beam trajectory can be perfectly corrected by the electron beam based alignment, coherent photon beam based alignment will be a complementary tool.

Figure 7 gives the spatial profile of coherent photon beam when the electron beam enters a single radiator segment with a transverse offset or angle; the radiator resonant wavelength is set to be less than the coherent photon beam wavelength, i.e., $49.49 \mathrm{~nm}$ in our case. The beam trajectory information can be obtained from the projection of the central part of spatial distribution. As shown by the first column plots in Fig. 7, because of an initial electron beam offset in the $x$ plane, the center of the coherent photon beam ring deviates from the undulator axis in horizontal, however, the projection amplitude does not change due to the relatively wide good field region of undulator in the $x$ plane. The phenomenon due to the beam offset in the $y$ plane could be explained by Eq. (3) induced undulator parameters $K$ enhancement. A tilted electron beam trajectory mainly changes the definition of the observation angle $\theta$ in Eq. (2), and thus introduces the variation shown in the last two columns.

It is worth stressing that inhomogeneity in the spatial distribution of the seed laser will significantly affect the transverse bunching and so affect the spatial distribution of the coherent photon beam. In order to generate a uniform

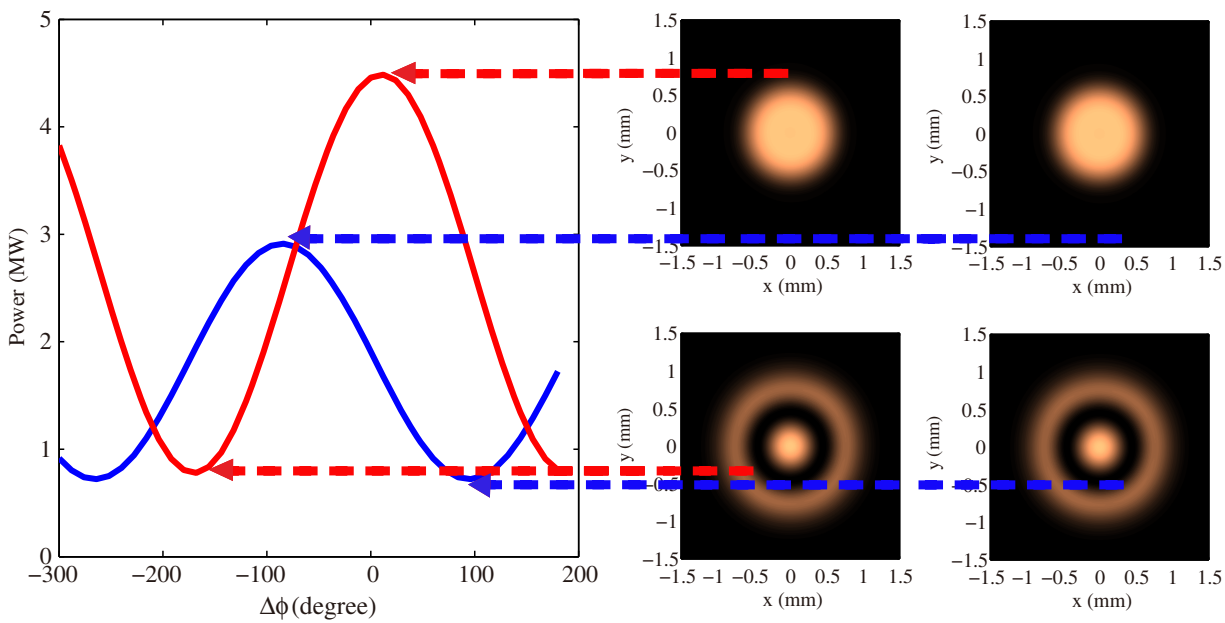

FIG. 6. Coherent photon beam spatial distribution of two successive undulator segments (blue: tuned to a wavelength of 49.49 nm; red: tuned to a wavelength of $49.84 \mathrm{~nm}$; spatial profile from one individual undulator segment can be seen in Fig. 3) for the best phase difference (the right upper two plots) and the worst phase difference (the right lower two plots). 

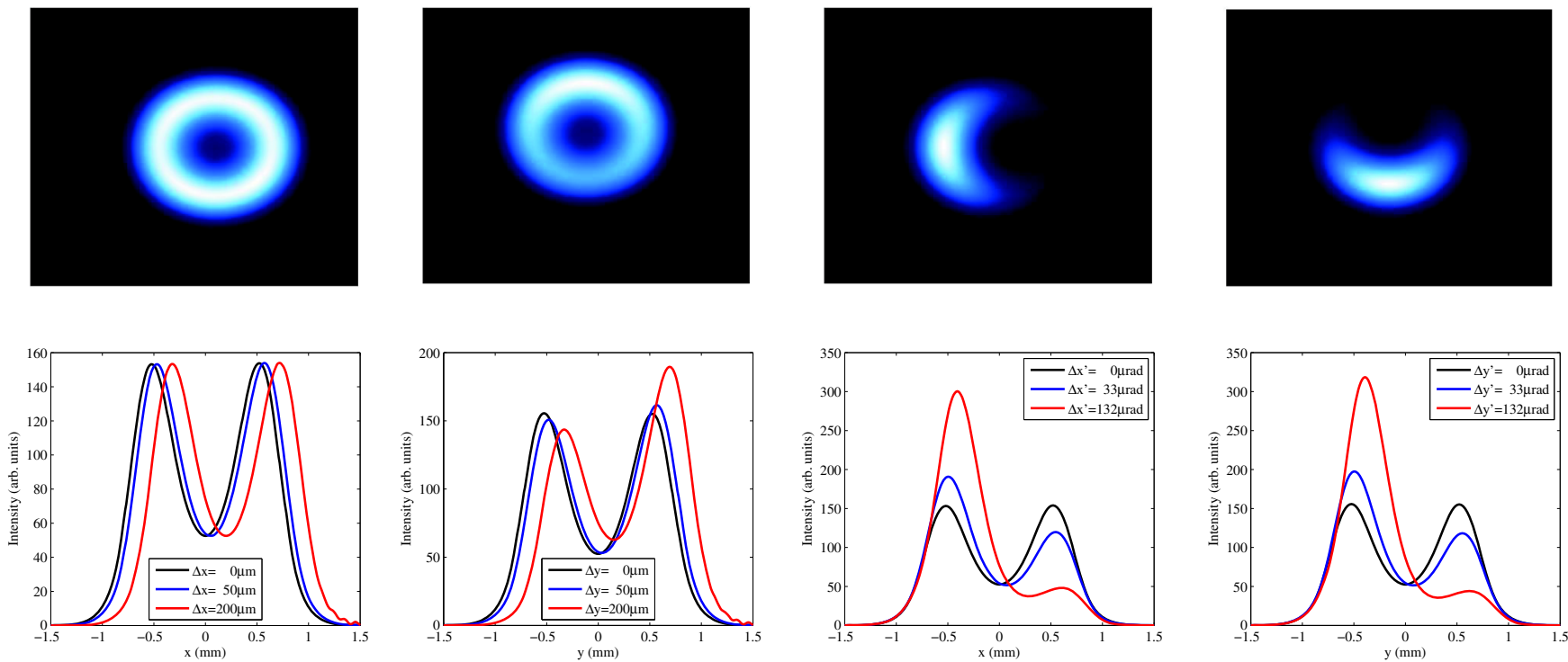

FIG. 7. The spatial distributions (the upper) and the central part projection (the lower) of the coherent photon beam with controlled electron beam offset and tilted injection with respect to undulator axis. Spatial distributions in the upper row correspond to the red lines in the plots in the lower row.
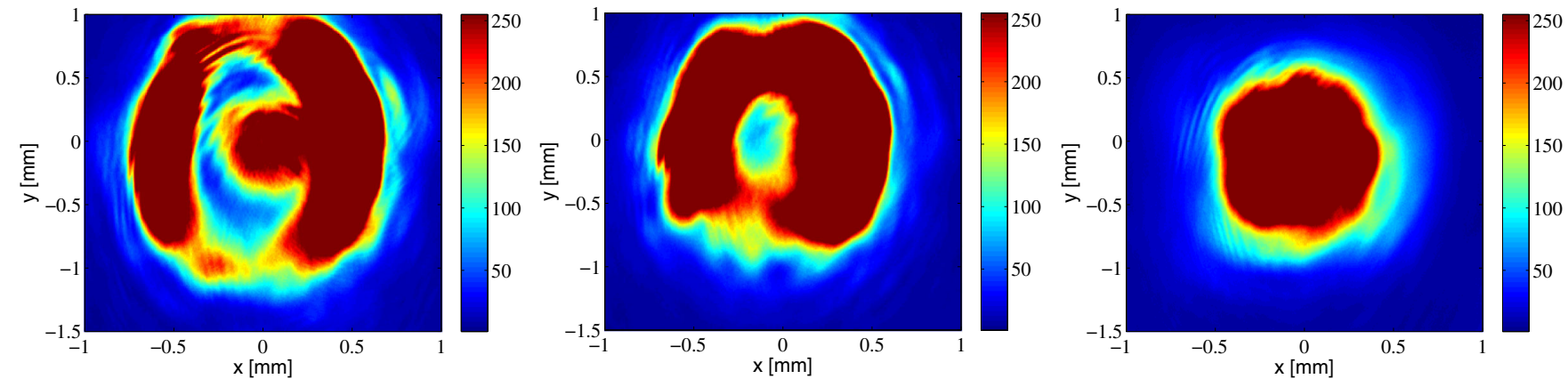

FIG. 8. Spatial profiles of the $400 \mathrm{~nm}$ coherent photon beam observed on the CCD camera downstream of the radiator.

transverse modulation, the spot size of the seed laser should be chosen to be much larger than that of the electron beam.

\section{Experiments at SDUV-FEL}

The SDUV-FEL is a multipurpose test facility for novel FEL principle studies, where echo-enabled harmonic generation FEL [26], crossed-planar undulators [46] and two-stage HGHG [47-49] have been successfully demonstrated. During the experiment of coherent photon beam diagnostics, the beam energy of SDUV-FEL was set to be $148 \mathrm{MeV}$. The seed laser comes from a commercial Ti-Sa system, which can provide up to several tens of micro-Joule energy with about $100 \mathrm{fs}$ pulse duration and $800 \mathrm{~nm}$ central wavelength. The modulator, a $10 \times 50 \mathrm{~mm}$ period length permanent magnet undulator, was set to fulfill the resonant conditions with beam energy and the seed laser. The radiator consisted of two segments of $1.6 \mathrm{~m}$ long permanent magnet undulators with $40 \mathrm{~mm}$ period length, and the magnetic gap of the radiator was tuned to be $16.5 \mathrm{~mm}$ for resonant at the 2nd harmonic of the seed laser, i.e., $400 \mathrm{~nm}$. A charge-coupled device (CCD) camera located downstream of the radiator was utilized for monitoring the spatial distribution of the coherent $400 \mathrm{~nm}$ photon beam.

Figure 8 illustrates the spatial distributions of the $400 \mathrm{~nm}$ coherent radiation pulses when adjusting the magnetic gap of the radiator, where the ring shape and core shape coherent photon beam profiles were both observed on the CCD camera. This phenomenon is successfully used to optimize the radiator gap to fit the resonant condition. It was found that the composed radiation intensity was maximized when the spatial distributions of the coherent photon beams from two single radiators transferred from a ring to a core profile, and transversely overlapped with each other.

\section{CONCLUSIONS}

With the fully coherent properties and the intrinsic amplification advantages of the output radiation from a 
single radiator segment in a seeded FEL, in the absence of a crystal based monochromator, a coherent photon beam based method is proposed to optimize the electron beam trajectory, to verify the undulator magnetic gap, and to adjust the phase match between two undulator segments. The principle of the proposed method is illustrated with parameters of Dalian coherent light source, a seeded extreme ultraviolet FEL user facility. Moreover, preliminary experimental results at the Shanghai deep ultraviolet FEL test facility are presented. It is worth stressing that all the diagnostics in this paper are based on the spatial analysis of coherent photon beam. If combined with the intensity investigation of coherent photon beam, this novel method will provide an effective and a complementary way for commissioning and alignment of the FEL undulator system, independently from the electron beam based procedures.

\section{ACKNOWLEDGMENTS}

This work is supported by the National Natural Science Foundation of China (No. 11175240, No. 11205234, No. 11322550, and No. 11475250) and the Major State Basic Research Development Program of China (No. 2011CB808300).

[1] W. Ackermann et al., Nat. Photonics 1, 336 (2007).

[2] P. Emma, R. Akre, J. Arthur et al., Nat. Photonics 4, 641 (2010).

[3] T. Ishikawa et al., Nat. Photonics 6, 540 (2012).

[4] E. Allaria et al., Nat. Photonics 7, 913 (2013).

[5] H. N. Chapman, P. Fromme, A. Barty et al., Nature (London) 470, 73 (2011).

[6] J. M. Glownia, Opt. Express 18, 17620 (2010).

[7] H. X. Deng, M. Zhang, D. Gu, B. Liu, Q. Gu, and D. Wang, Chin. Phys. C 38, 028101 (2014).

[8] J. Yan, M. Zhang, and H. X. Deng, Nucl. Instrum. Methods Phys. Res., Sect. A 615, 249 (2010).

[9] E. Allaria, C. Callegari, D. Cocco, W. M. Fawley, M. Kiskinova, C. Masciovecchio, and F. Parmigiani, New J. Phys. 12, 075002 (2010).

[10] B. Faatz, N. Baboi, V. Ayvazyan et al., Nucl. Instrum. Methods Phys. Res., Sect. A 635, S2 (2011).

[11] M. Altarelli et al., XFEL: The European X-Ray Free-Electron Laser, Technical Design Report (DESY, Hamburg, 2006).

[12] R. Ganter et al., PSI Report No. 10-04, 2010.

[13] J.-H. Han, H.-S. Kang, and I. S. Ko, in Proceedings of the 3rd International Particle Accelerator Conference, New Orleans, LA, 2012 (IEEE, Piscataway, NJ, 2012), p. 1735.

[14] B. Faatz, Report No. TESLA-FEL 2001-04, 2001.

[15] P. Castro, Report No. TESLA-FEL 1997-04, 1997.

[16] P. Emma, LCLS Technical Notes 00-14, 2000.

[17] M. Tischer, P. Ilinski, U. Hahn, J. Pflüger, and H. SchulteSchrepping, Nucl. Instrum. Methods Phys. Res., Sect. A 483, 418 (2002).
[18] T. Tanaka, S. Goto, T. Hara, T. Hatsui, H. Ohashi, K. Togawa, M. Yabashi, and H. Tanaka, Phys. Rev. ST Accel. Beams 15, 110701 (2012).

[19] C. Ozkan, W. Freund, J. Rehanek, I. Zizak et al., in X-Ray Free-Electron Lasers: Beam Diagnostics, Beamline Instrumentation, and Applications, SPIE Proceedings Vol. 8504 (SPIE-International Society for Optical Engineering, Bellingham WA, 2012), DOI: 10.1117/12.929755.

[20] R. Bonifacio, C. Pellegrini, and L. Narducci, Opt. Commun. 50, 373 (1984).

[21] J. Andruszkow, B. Aune, V. Ayvazyan et al., Phys. Rev. Lett. 85, 3825 (2000).

[22] W. Ackermann, G. Asova, V. Ayvazyan et al., Nat. Photonics 1, 336 (2007).

[23] L. Yu, L. DiMauro, A. Doyuran et al., Phys. Rev. Lett. 91, 074801 (2003).

[24] G. Lambert, T. Hara, D. Garzella et al., Nat. Phys. 4, 296 (2008).

[25] D. Xiang, E. Colby, M. Dunning et al., Phys. Rev. Lett. 105, 114801 (2010).

[26] Z. T. Zhao, D. Wang, J. H. Chen et al., Nat. Photonics 6, 360 (2012).

[27] A. Azima, J. Bödewadt, F. Curbis et al., in Proceedings of the International Particle Accelerator Conference, Kyoto, Japan (ICR, Kyoto, 2010), pp. 2161-2163.

[28] J. Amann, W. Berg, V. Blank et al., Nat. Photonics 6, 693 (2012).

[29] L. H. Yu, Phys. Rev. A 44, 5178 (1991).

[30] G. Stupakov, Phys. Rev. Lett. 102, 074801 (2009).

[31] D. Xiang and G. Stupakov, Phys. Rev. ST Accel. Beams 12, 030702 (2009).

[32] H. Deng and C. Feng, Phys. Rev. Lett. 111, 084801 (2013).

[33] J. Feldhausa, E. L. Saldinb, J. R. Schneidera, E. A. Schneidmiller, and M. V. Yurkov, Opt. Commun. 140, 341 (1997).

[34] G. Geloni, V. Kocharyan, and E. Saldin, J. Mod. Opt. 58, 1391 (2011).

[35] Z. T. Zhao, Z. M. Dai, X. F. Zhao, D. K. Liu, Q. G. Zhou, D. H. He, Q. K. Jia, S. Y. Chen, and J.P. Dai, Nucl. Instrum. Methods Phys. Res., Sect. A 528, 591 (2004).

[36] E. L. Saldin, E. A. Schneidmiller, and M. V. Yurkov, Nucl. Instrum. Methods Phys. Res., Sect. A 539, 499 (2005).

[37] C. Feng, T. Zhang, J. Chen, H. Deng, M. Zhang, X. Wang, B. Liu, T. Lan, D. Wang, and Z. Zhao, Phys. Rev. ST Accel. Beams 14, 090701 (2011).

[38] H. X. Deng, C. Feng, B. Liu, D. Wang, X.-T. Wang, and M. Zhang, Chin. Phys. Lett. 28, 124101 (2011).

[39] S. Reiche, Nucl. Instrum. Methods Phys. Res., Sect. A 429, 243 (1999).

[40] M. R. Howells and B. M. Kincaid, The Properties of Undulator Radiation, edited by F.S. Schlachter and F. Wuilleumier (Kluwer Academic Publishers, Dordrecht, 1994), pp. 315-358.

[41] K. Floettmann, ASTRA User's Manual, available at http:// www.desy.de/mpyflo/Astra_dokumentation (1999).

[42] M. Borland, Phys. Rev. ST Accel. Beams 4, 070701 (2001).

[43] X. J. Wang, H. P. Freund, and D. Harder, Phys. Rev. Lett. 103, 154801 (2009). 
[44] G. Dattoli, S. Pagnutti, P. L. Ottaviani, and V. Asgekar, Phys. Rev. ST Accel. Beams 15, 030708 (2012).

[45] Y. Jiao, J. Wu, Y. Cai, A. W. Chao, W. M. Fawley, J. Frisch, Z. Huang, H.-D. Nuhn, C. Pellegrini, and S. Reiche, Phys. Rev. ST Accel. Beams 15, 050704 (2012).

[46] H. Deng et al., Phys. Rev. ST Accel. Beams 17, 020704 (2014).
[47] H.X. Deng and Z. M. Dai, Chin. Phys. C 32, 236 (2008).

[48] C. Feng, M. Zhang, G. Lin, Q. Gu, H. Deng, J. Chen, D. Wang, and Z. Zhao, Chin. Sci. Bull. 57, 3423 (2012).

[49] B. Liu et al., Phys. Rev. ST Accel. Beams 16, 020704 (2013). 